\title{
Viscometric and Light Scattering Study in Dimethylacetamide of Linear Segment Polyurethane, Polymerized with Methylene Bis(4-phenyl isocyanate), Polytetramethylene Glycol and Ethylene Diamine
}

\author{
Kenji KaMIDE, Akira KIgUCHI, and Yukio MiYAZAKI \\ Fundamental Research Laboratory of Fibers and Fiber-Forming Polymers, \\ Asahi Chemical Industrý Company, Ltd., Takatsuki, Osaka 569, Japan
}

(Received May 16, 1986)

\begin{abstract}
An attempt was made to determine the unperturbed chain dimension $A$ of a segment polyurethane, prepared from polytetramethylene glycol (the number-average molecular weight 1500 ), methylene bis(4-phenyl isocyanate) and ethylenediamine. A whole polymer was fractionated by successive solution fractionation using dimethylacetamide (DMAc) as a solvent and methylalcohol as a precipitant into 23 fractions. Linearity of the segment polyurethane chain was confirmed by NMR and the solution viscosity change by addition of $n$-butylamine. The dilute solution properties of eight fractions in DMAc were investigated by the light scattering and viscometry at $25^{\circ} \mathrm{C}$. The Mark-Houwink-Sakurada equation for the solution in DMAc at $25^{\circ} \mathrm{C}$ was $[\eta]=0.101 \bar{M}_{w}^{0.59}\left(\mathrm{~cm}^{3} \mathrm{~g}^{-1}\right)\left([\eta]\right.$; limiting viscosity number, $\bar{M}_{w}$; the weight-average molecular weight). $A$ was found to be $1.02 \times 10^{-8} \mathrm{~cm}$ for the solution. No significant solvation was observed by adiabatic compressibility measurement.
\end{abstract}

KEY WORDS Segment Polyurethane / Unperturbed Chain Dimension / Light Scattering / Limiting Viscosity Number / Mark-Houwink-Sakurada Equation / Solvation /

Linear segment polyurethane (LSP) generally consists of so-called hard- and softsegments and the hydrogen bond is often formed between the urea and urethane groups in the hard- and soft-segments. LSP is commonly used as the starting material of highly elastic fibers because the hard segments highly crystallize to give a network cross point and as a result, the polymer exhibits excellent rubber elasticity. Some studies were made on the super structure and physical properties of the high elastic segment polyurethane fibers. ${ }^{1}$ Surprisingly, up to now the molecular properties of this kind of polymer were not attempted to investigate systematically with a few exceptions. Sato ${ }^{2}$ obtained the relations between the limiting viscosity number $[\eta]$ and molecular weight by the sedimenta- tion velocity method, and Lyman ${ }^{3}$ synthesized a linear polyurethane by polyaddition of equimolar amount of ethylene glycol and methylene bis(4-phenyl isocyanate) (MDI) and obtained the rather scattered log-log plot of $[\eta]$ and the weight-average molecular weight $\bar{M}_{w}$ by light scattering for five unfractionated samples in $N, N$-dimethylformamide (DMF) (Figure 1 of ref 3), for which he did not estimate the Mark-Houwink-Sakurada (MHS) equation: $[\eta]=K_{\mathrm{m}} \bar{M}_{w}^{a}$, were $K_{\mathrm{m}}$ and $a$ are constants characteristic of a given combination of polymer and solvent at a given temperature. Using Lyman's plot, Beachell and Peterson ${ }^{4}$ evaluated MHS equation in DMF and on the basis of this equation they obtained the MHS equations in DMF/acetone mixtures at $25^{\circ} \mathrm{C}$. They also estimated the 
unperturbed chain dimension $\mathrm{A}$ from $K_{\mathrm{m}}$ at $a=0.5 \quad(\mathrm{DMF}$-acetone $=71: 29, \mathrm{v} / \mathrm{v})$. Here, note that the reliability of their conclusions depends basically on that of Lyman's original plot.

In this article, an attempt was made to investigate by the viscometry and light scattering the dilute solution properties of a linear segment polyurethane, polymerized with methylene bis(4-phenyl isocyanate) (MDI), polytetramethylene glycol and ethylene diamine.

\section{EXPERIMENTAL}

\section{Sample}

The prepolymer as soft segments was prepared by reacting $0.1 \mathrm{~mol}$ of polytetramethylene glycol (PTG, the number-average molecular weight $\bar{M}_{n}$ is 1500 ) with $0.15 \mathrm{~mol}$ of MDI (the molecular weight is 250 ) at $80^{\circ} \mathrm{C}$ for $120 \mathrm{~min}$. The polymer was dissolved in 1 liter of $N, N$-dimethylacetamide (DMAc) and the segment polyurethane was polymerized by adding an equivalent amount of ethylene diamine to the prepolymer-DMAc solution at $40^{\circ} \mathrm{C}$ for $60 \mathrm{~min}$. The limiting viscosity number [ $\eta$ ] of this polymer was $108 \mathrm{mlg}^{-1}$ in DMAc at $25^{\circ} \mathrm{C}$ and the viscosity-average molecular weight $\bar{M}_{v}$ was $13.6 \times 10^{4}$ as calculated from the MHS equation established here (see eq a).

The chemical structure of the soft and hard segments of the segment polyurethane synthesized here are illustrated in Figure 1.

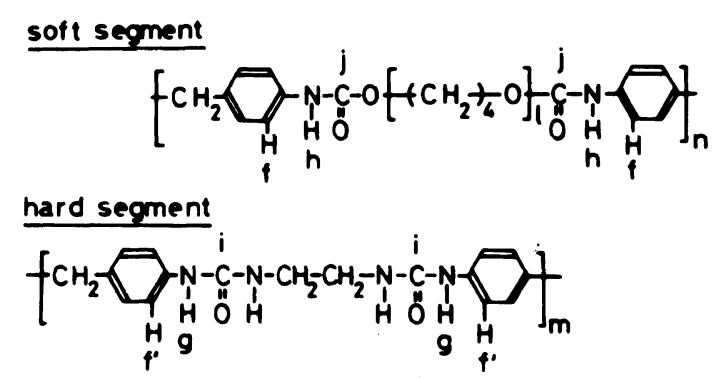

Figure 1. Molecular structure of the segment polyurethane.

\section{Solvents}

Guaranteed reagent grade DMAc, manufactured by Kishida Chemicals (Osaka), was used as received for fractionation, light scattering, solution viscosity and solvation measurements. For polymerization experiments, DMAc was distilled under reduced atmosphere and a middle portion of the distillate was used.

\section{Fractionation}

Successive solution fractionation (SSF) was applied to a whole polymer using DMAc as a good solvent and methyl alcohol as a precipitant and 23 fractions were separated. The starting polymer concentration was 2.5 $\mathrm{wt} \%$ and temperature was $25^{\circ} \mathrm{C}$.

\section{Light Scattering}

Light scattering of the DMAc solution was measured at $25^{\circ} \mathrm{C}$ with unpolarized incident light at a wave-length $\lambda_{0}$ of $546 \mathrm{~nm}$ in a scattering angle $\theta$ range $30 \sim 150^{\circ}$. A FICA photogoniodiffusiometer 42000 was utilized. 0.5 gram of the polymer was dissolved in 100 $\mathrm{ml}$ of DMAc at $25^{\circ} \mathrm{C}$ for $2 \mathrm{~h}$ to give the crude solution, which was centrifuged at $49200 \mathrm{~g}$, using a Hitachi ultracentrifuge model 55P7 , at $20^{\circ} \mathrm{C}$ for $70 \mathrm{~min}$. The supernatant phase of the solution was isolated and diluted with pure DMAc to prepare the sample solutions $(30 \mathrm{ml}$ each) with four different concentrations. Immediately before the light scattering measurements, the solution was filtered with a poly-4-fluoroethylene membrane (trade mark, Fluoro pore FP-010, manufactured by Sumitomo Denko Co., average pore size $0.10 \mu \mathrm{m})$, directly into the measuring cell. The specific refractive index increment $\mathrm{d} n$ / $\mathrm{d} C$ for this system was evaluated to be 0.062 $\mathrm{cm}^{-3} \mathrm{~g}^{-1}$ on a Shimadzu differential refractometer model DR-4 at $\lambda_{0}$ of $546 \mathrm{~nm}$. The data obtained were analyzed according to Zimm's procedure to evaluate the weightaverage molecular weight $\bar{M}_{w}, z$-average radius of gyration $\left\langle S^{2}\right\rangle_{z}^{1 / 2}$ and second virial 
coefficient $A_{2}$.

\section{Solution Viscosity}

The solution viscosity was measured in DMAc using a modified Ubbelohde suspension type viscometer at $25 \pm 0.01^{\circ} \mathrm{C}$. The limiting viscosity number $[\eta]$ was obtained by Huggins plot.

\section{Nuclear Magnetic Resonance Spectrum}

Nuclear magnetic resonance (NMR) spectra were obtained for the fractions in a deuterated dimethyl sulfoxide (DMSO- $d_{6}$ ) and tetrachlorocarbon mixture $(1: 2, \mathrm{v} / \mathrm{v})$ (the polymer concentration, $5 \mathrm{wt} \%$ ) from a JOEL FX-400 Pulse-Fourier Transform NMR spectrometer at $50^{\circ} \mathrm{C}$. TMS was used as the reference.

\section{Adiabatic Compressibility}

The ultrasonic velocity $v$ of the solutions in DMAc at a concentration of $1 \sim 8 \mathrm{~g} / 100 \mathrm{~g}$ DMAc was measured with a Nusonics model 6080 concentration analyzer, operating at $5 \mathrm{MHz}$ and $25 \pm 0.005^{\circ} \mathrm{C}$. The detailed measuring procedure has already been described elsewhere by Kamide and Saito. ${ }^{5} v$ was converted with help of the density of the solution to the adiabatic compressibility $\beta$, from which the weight of the solvated solvent per gram of polymer $N_{\mathrm{s}}$ was calculated by Passynsky equation. ${ }^{6}$

\section{Elementary Analysis}

Elementary analysis of the polymer samples was made by CHN analyzer model NT-2 and model NOX-10, manufactured by Yanagimoto Manufacturing Co. (Kyoto).

\section{RESULTS AND DISCUSSION}

The elementary analysis for two fractions, PU 1-2 and PU $1-22$ with $\bar{M}_{w}=3.16 \times 10^{4}$ and $35.0 \times 10^{4}$ found: C, $65.9 \mathrm{wt} \% ; \mathrm{H}, 9.5 \mathrm{wt} \%$; $\mathrm{O}, 20.6 \mathrm{wt} \% ; \mathrm{N}, 4.2 \mathrm{wt} \%$, and $\mathrm{C}, 65.9 \mathrm{wt} \%$; $\mathrm{H}, 10.4 \mathrm{wt}^{\circ} \%$; O $21.2 \mathrm{wt}^{\mathrm{o}} \%$; N, $4.0 \mathrm{wt}^{\circ} \%$, re- spectively. It is clear that the contents of $\mathrm{C}, \mathrm{H}, \mathrm{O}$, and $\mathrm{N}$ of these samples are practically the same.

Analyzing the ${ }^{1} \mathrm{H}$ NMR spectrum assigned for $\mathrm{NH}$ proton (denoted by $\mathrm{g}$ in Figure 1) in urea group of the hard segment, neighboring aromatic proton (denoted by $\mathrm{f}^{\prime}$ in Figure 1), $\mathrm{NH}$ proton (denoted by $\mathrm{h}$ in Figure 1) in. urethane group of the soft segment and neighboring aromatic proton (denoted by $\mathrm{f}$ in Figure 1), we estimated the ratio $n / m$ of the polymer, where $n$ and $m$ are the numbers of repeating units constituting a soft segment and a hard segment, respectively.

Figure 2 shows ${ }^{1} \mathrm{H}$ NMR and ${ }^{13} \mathrm{C}$ NMR spectrum of the two fractions with largely different $\bar{M}_{w}$. Assignment of the peaks has already been given by $\mathrm{Okuto}^{7}$ and $\mathrm{Kaji}^{8}{ }^{8}$ The symbols $g, \mathrm{f}^{\prime}, \mathrm{h}, \mathrm{f}, \mathrm{i}$, and $\mathrm{j}$ in Figure 1 are $\mathrm{NH}$ proton $(8.17 \mathrm{ppm})$ in urea group of the hard segment, its neighboring aromatic proton $(7.25 \mathrm{ppm}), \mathrm{NH}$ proton $(9.06 \mathrm{ppm})$ in the urethane group of the soft segment, its neighboring aromatic proton (7.32 ppm), carbonyl carbon $(155 \mathrm{ppm})$ in the urea group of the hard segment and the carbonyl carbon (153 ppm) in the urethane group of the soft segment, respectively.

The peak intensities $p$ corresponding to $f$, $\mathrm{f}^{\prime}, \mathrm{g}, \mathrm{h}, \mathrm{i}$, and $\mathrm{j}$ are evaluated from Figure 2.

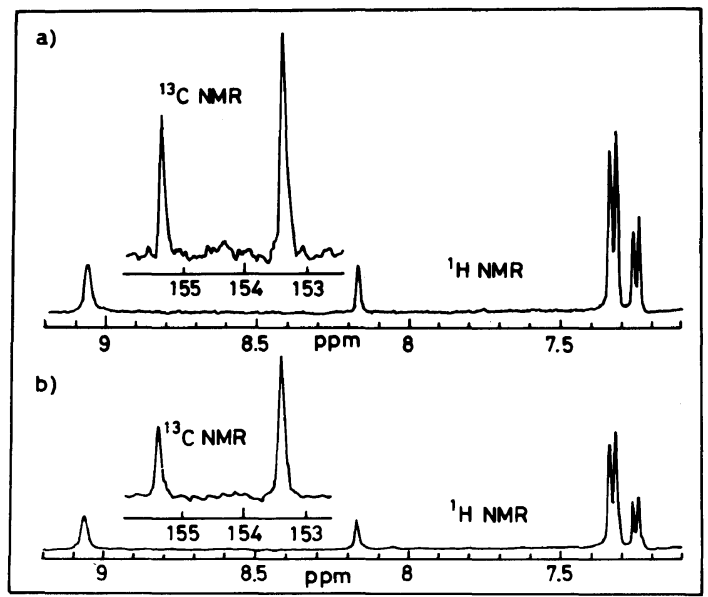

Figure 2. ${ }^{1} \mathrm{H} \mathrm{NMR}$ and ${ }^{13} \mathrm{C} N \mathrm{NMR}$ spectrum of the fractions PU 1-2 (a) and PU 1-22 (b). 
Table I. The ratio $n / m$ of repeating units constituting soft and hard segments

\begin{tabular}{lcccc}
\hline & & \multicolumn{3}{c}{$n / m$} \\
\cline { 3 - 5 } Sample & $\bar{M}_{w} \times 10^{-4}$ & $p_{\mathrm{f}} / p_{\mathrm{f}^{\prime}}$ & $p_{\mathrm{h}} / p_{\mathrm{g}}$ & $p_{\mathrm{i}} / p_{\mathrm{j}}$ \\
\hline PU 1-2 & 3.16 & 2.18 & 2.00 & 1.94 \\
PU 1-22 & 35.0 & 2.33 & 2.00 & 2.24 \\
\hline
\end{tabular}

Table I contains the ratio of intensities $p_{\mathrm{f}} /$ $p_{\mathrm{f}^{\prime}}, p_{\mathrm{h}} / p_{\mathrm{g}}$ and $p_{\mathrm{i}} / p_{\mathrm{j}}$. These ratios should give the ratio of $n / m$ and they are almost the same (=2.1), irrespective of $\bar{M}_{w}$, which is not far from the predicted value $(=2.0)$ of $n / m$ from the polymerization conditions.

The elementary analysis and ${ }^{1} \mathrm{H}$ NMR and ${ }^{13} \mathrm{C}$ NMR spectroscopic data show definitely that the compositions of the hard and soft segments are constant, independent of the molecular weight.

It was found that for polyurethane having biuret or allophanate binding due to a trifunctional branching structure, $[\eta]$ of the solution in DMAc decreases by (1) adding primary amine at $50^{\circ} \mathrm{C}$ or by (2) heating the solution at $100^{\circ} \mathrm{C}$, approaching within $2 \mathrm{~h}$ (case (1)) or several h (case (2)) to an asymptotic value. ${ }^{9}$ The above change in $[\eta]$ can be attributed to the molecular weight lowering during the process of conversion of the branched molecule to the linear molecule with the breakage of biuret or allophanate bindings in the polymer by amines added or brought about by thermal decomposition material of DMAc (dimethylamine). The mechanism of amine decomposition of biuret, allophanate bindings was studied by Okuto. $^{7}$ No significant decrease in $[\eta]$ of any fractions used here was observed by amine addition or heating the solution. In addition, no carbon peak assigned for biuret or allophanate binding was detected in the ${ }^{13} \mathrm{C}$ NMR spectrum ${ }^{8}$ and the peak intensity of $\mathrm{NH}$ proton in urea or the urethane group did not change significantly by adding amines to the sample solutions in DMAc. Then, we can conclude that the segment polyurethane prepared in this study should be a linear polymer.

Hard segment of segment polyurethane is an alternative copolymer of MDI and ethylenediamine and the soft segment is an alternative copolymer of MDI and PTG. Then, segment polyurethane is an alternative block copolymer of hard and soft segments (Figure 1). The molecular weight of the hard segment consisting of segment polyurethane is expected, more or less, not to be rigorously uniform, although the chemical composition is the same and also, PTG in the soft segment has unavoidably the molecular weight distribution, resulting in heterogeneity of the molecular weight and the chemical composition of the soft segment. Consequently, the chemical composition distribution of the segment polyurethane chains used here should be taken into consideration when light scattering is carried out for the solution. ${ }^{10}$ In other words, in order to determine $\bar{M}_{w}$ of segment polyurethane, the light scattering measurements should be carried out in several kinds of solvents having different refractive indices. Unfortunately, adequate solvents for the light scattering are only DMAc and DMF, whose refractive indices are not significantly different (i.e., 1.428 for the former and 1.435 for the latter). The apparent weightaverage molecular weight $\bar{M}_{w, \text { a }}$, the apparent radius of gyration $\left\langle S^{2}\right\rangle_{z, \mathrm{a}}^{1 / 2}$ and the apparent second virial coefficient $A_{2, \mathrm{a}}$ were evaluated in DMAc solution in this paper. But as described before, we observed for polyurethane fractions with different $[\eta]$ in DMAc a constant average chemical composition and the same $\mathrm{d} n / \mathrm{d} C$ in DMAc. This means that we can use, to the first approximation, $\bar{M}_{w, \mathrm{a}}$ of the fractions as a parameter representing $\bar{M}_{w}$ and to avoid complexity, we denote hereafter $\bar{M}_{w, \mathrm{a}}$, $\left\langle S^{2}\right\rangle_{z, \mathbf{a}}^{1 / 2}$, and $A_{2, \mathrm{a}}$ simply, as $\bar{M}_{w},\left\langle S^{2}\right\rangle_{z}^{1 / 2}$ and $A_{2}$, respectively.

Table II summarizes $[\eta], \bar{M}_{w},\left\langle S^{2}\right\rangle_{z}^{1 / 2}$ and $A_{2}$ of the fractions in DMAc at $25^{\circ} \mathrm{C}$. Figure 


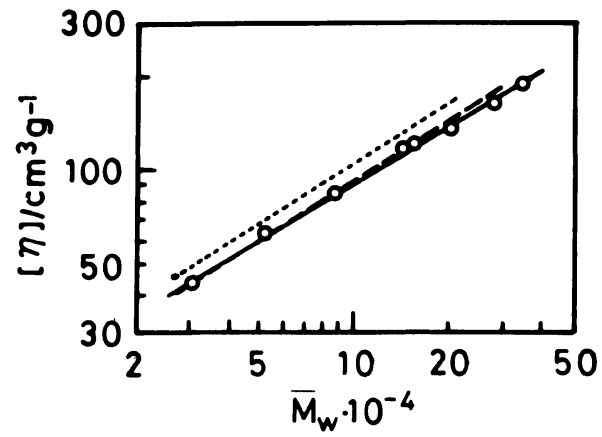

Figure 3. Log-log plots of limiting viscosity number versus the weight-average molecular weight $\bar{M}_{w}$ for the segment polyurethane in DMAc at $25^{\circ} \mathrm{C}(\mathrm{O})$; dotted line, the polymer $\mathrm{B}^{2}$; broken line, polymer $\mathrm{C}^{2}$ in $\mathrm{DMF}$ at $25^{\circ} \mathrm{C}$.

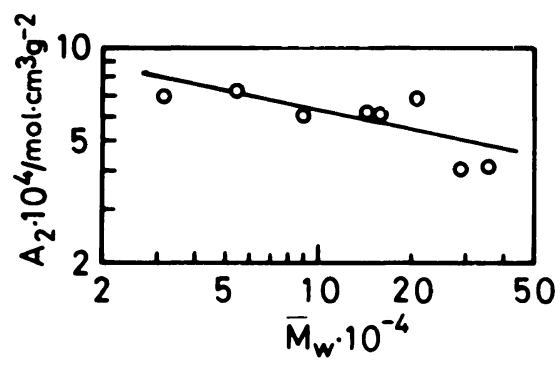

Figure 4. Molecular weight dependence of the second virial coefficient $A_{2}$ for the segment polyurethane in DMAc at $25^{\circ} \mathrm{C}$.

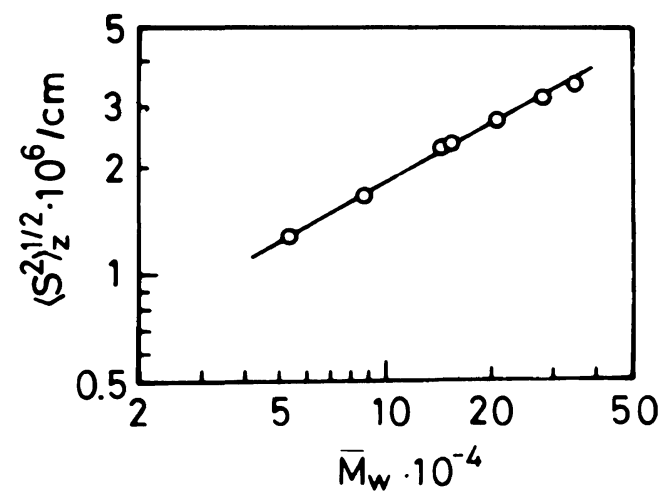

Figure 5. Plot of the radius of gyration $\left\langle S^{2}\right\rangle_{z}^{1 / 2}$ as a function of molecular weight for the segment polyurethane in DMAc at $25^{\circ} \mathrm{C}$.

3,4 , and 5 show the $\log -\log$ plots of $[\eta]$, $\left\langle S^{2}\right\rangle_{z}^{1 / 2}$ and $A_{2}$ versus $\bar{M}_{w}$, respectively.

Using the least-squares method, we ob-
Table II. Results of light scattering and viscosity measurements on segment polyurethane fractions in DMAc at $25^{\circ} \mathrm{C}$

\begin{tabular}{cccccc}
\hline \multirow{2}{*}{$\begin{array}{c}\text { Sample } \\
\text { code }\end{array}$} & \multicolumn{1}{c}{$[\eta]$} & & $A_{2} \times 10^{4}$ & & \multicolumn{2}{c}{$\left\langle S^{2}\right\rangle_{z}^{1 / 2} \times 10^{8}$} \\
\cline { 6 - 6 } \cline { 5 - 6 } $\mathrm{cm}^{3} \mathrm{~g}^{-1}$ & $\bar{M}_{w} \times 10^{-4}$ & & $\mathrm{~cm}^{3} \mathrm{~mol} \mathrm{~g}^{-2}$ & & $\mathrm{~cm}$ \\
\hline PU 1-2 & 43 & 3.16 & 7.00 & & - \\
PU 1-5 & 65 & 5.36 & 7.38 & & $12 \dot{8}$ \\
PU 1-7 & 84 & 8.94 & 6.07 & & 167 \\
PU 1-10 & 117 & 14.3 & 6.19 & & 227 \\
PU 1-12 & 120 & 15.4 & 6.10 & & 231 \\
PU 1-15 & 135 & 20.8 & 6.89 & & 266 \\
PU 1-18 & 161 & 28.1 & 4.00 & & 304 \\
PU 1-22 & 184 & 35.0 & 4.15 & 339 \\
\hline
\end{tabular}

tain

$$
\begin{gathered}
{[\eta]=0.101 \bar{M}_{w}^{0.59}\left(\mathrm{~cm}^{3} \mathrm{~g}^{-1}\right)} \\
\left\langle S^{2}\right\rangle_{z}^{1 / 2}=0.358 \times 10^{-8} \bar{M}_{w}^{0.54}(\mathrm{~cm}) \\
A_{2}=7.22 \times 10^{-3} \bar{M}_{w}^{-0.21}\left(\mathrm{~cm}^{3} \mathrm{~mol} \mathrm{~g}^{-2}\right)
\end{gathered}
$$

Figure 3 also includes literature data by Sato. ${ }^{2}$ The MHS equations on the polymer B (diol, polycaprolactone; $\bar{M}_{n}=1300$; prepolymerization, MDI : diol $=2: 1 \mathrm{~mol} / \mathrm{mol}$ in bulk for $110 \mathrm{~min}$ at $75^{\circ} \mathrm{C}$; polymerization, ethylenediamine $:$ prepolymer $=1: 1 \mathrm{~mol} / \mathrm{mol}$ in DMAc at $40^{\circ} \mathrm{C}$ ) and the polymer $\mathrm{C}$ (diol, polycaprolactone $\bar{M}_{n}=2800$ and other conditions. are the same as polymer B), both obtained by Sato $^{2}$ are shown by dotted and broken lines, respectively.

The exponent in eq $b(0.54)$ is consistent with that in eq a $(0.59)$ when the partially draining effect is neglected. $A_{2}$ lies between $0.4 \times 10^{-3}-0.7 \times 10^{-3} \mathrm{~mol} \mathrm{~g}^{-2}, \quad$ considerably smaller than those of typical polymers dissolved in good solvents. Judging from the magnitude of the exponents in eq $a$ and $b$ and $A_{2}$, DMAc is concluded not to be a very good solvent.

The unperturbed chain dimension $A$ $\left(\equiv\left(\left\langle R^{2}\right\rangle_{0} / M\right)^{1 / 2},\left\langle R^{2}\right\rangle_{0}^{1 / 2}\right.$ is the unperturbed mean-square end-to-end distance of the polymer) was evaluated by the following method: 
Method $2 B$

$$
A \equiv 6^{1 / 2}\left(\frac{\left\langle S^{2}\right\rangle_{0}}{M}\right)^{1 / 2}=6^{1 / 2}\left(\frac{\left\langle S^{2}\right\rangle / \alpha_{\mathrm{s}}^{2}}{M}\right)^{1 / 2}
$$

$\alpha_{\mathrm{s}}$ is the linear expansion factor, calculated using the penetration function $\psi$ from $A_{2}, \bar{M}_{w}$ and $\left\langle S^{2}\right\rangle^{1 / 2}$ data. Here, Kurata-FukatsuSotobayashi-Yamakawa theory, ${ }^{11}$ connecting $\psi$ with the excluded volume parameter $z$ and the Fixman theory ${ }^{12}$ of the excluded volume effect were utilized.

\section{Method 2C (Baumann plot $)^{13}$}

$$
\left(\left\langle S^{2}\right\rangle / M\right)^{3 / 2}=A^{3} / 6^{3 / 2}+\left(1 / 4 \pi^{3 / 2}\right) B M^{1 / 2}
$$

where $B$ is a long-range interaction parameter.

Method 2E (Stockmayer-Fixman plot $)^{14}$

$$
[\eta] / M^{1 / 2}=K+2(3 / 2 \pi)^{3 / 2} \Phi(\infty) B M^{1 / 2}
$$

with

$$
\begin{gathered}
K=\Phi_{0}(\infty) A^{3} \\
\Phi_{0}(\infty)=2.87 \times 10^{23}
\end{gathered}
$$

Method $2 F$ (Kamide-Moore plot $)^{15}$

$$
\begin{gathered}
-\log K_{\mathrm{m}}+\log \left[1+2\left\{(a-0.5)^{-1}-2\right\}^{-1}\right] \\
=-\log K+(a-0.5) \log M_{0}
\end{gathered}
$$

where $M_{0}$ is the geometric average of upper and lower limits of $M$, in which the MHS equation is applied.

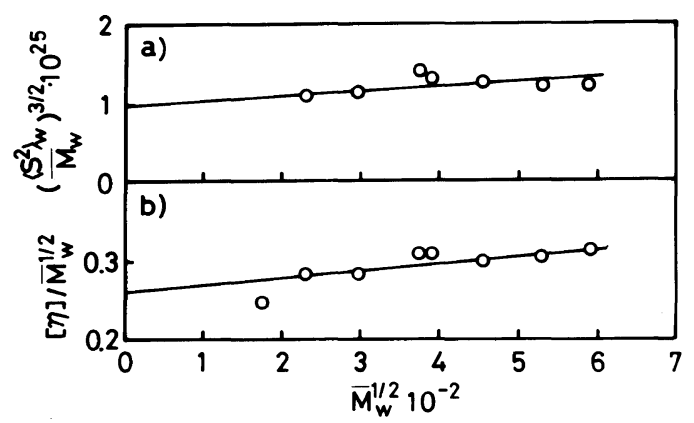

Figure 6. a) Baumann plot (method 2C) and b) Stockmayer-Fixman plot (method 2E) for the segment polyurethane in DMAc at $25^{\circ} \mathrm{C}$.
Figure 6 shows Baumann and Stockmayer -Fixman plots. The $A$ values estimated by method $2 \mathrm{~B}, 2 \mathrm{C}, 2 \mathrm{E}$, and $2 \mathrm{~F}$ are $1.07 \times 10^{-8}$, $1.05 \times 10^{-8}, 0.99 \times 10^{-8}$, and $0.97 \times 10^{-8} \mathrm{~cm}$, respectively and averaged to $1.02 \times 10^{-8} \mathrm{~cm}$.

The unperturbed chain dimension of a hypothetical chain with free internal rotation $A_{\mathrm{f}}$ was calculated through use of the relation:

$$
A_{\mathrm{f}}=\left[\frac{\bar{l}^{2}}{\bar{M}_{\mathrm{b}}}\{(1-\cos \theta) /(1+\cos \theta)\}\right]^{1 / 2}
$$

with

$$
\bar{l}=\frac{\sum l_{i} n_{i}}{\sum n_{i}}
$$

( $i=\mathrm{CH}_{2}-\mathrm{CH}_{2}, \quad \mathrm{CH}_{2}-\mathrm{O}, \quad \mathrm{CO}-\mathrm{O}, \quad \mathrm{CO}-\mathrm{NH}$, $\phi-\mathrm{CH}_{2}, \phi-\mathrm{NH}, \phi, \mathrm{NH}-\phi$; here, $\phi$ denote benzene ring)

$$
\bar{M}_{\mathrm{b}}=\frac{\Sigma M_{j} m_{j}}{\Sigma m_{j}}
$$

$\left(j=\mathrm{CH}_{2}, \mathrm{NH}, \mathrm{CO}, \mathrm{O}, \phi\right)$

$\bar{l}$ is the effective bond length, $l_{i}$, the bond length of the $i$ th kind of bonds and $n_{i}$ is the number of $i$ th kinds of bonds in a given chain fragment (in this case repeating unit). $\bar{M}_{\mathrm{b}}$ is the average molecular weight per unit skeletal bond, $m_{j}$ is the number of $j$ kinds of atomic groups and $M_{j}$ is the molecular weight of the $j$ kinds of atomic groups.

In Figure 1, a chain with $m=1, n=2$, and $l=21$ can be regarded as a repeating unit of the segment polyurethane investigated here. From the figure we obtain $n_{1}=127$, $l_{1}=1.54 \AA\left(\mathrm{CH}_{2}-\mathrm{CH}_{2}\right), n_{2}=86, l_{2}=1.43 \AA$ $\left(\mathrm{CH}_{2}-\mathrm{O}\right), n_{3}=4, l_{3}=1.34 \AA(\mathrm{CO}-\mathrm{O}), n_{4}=8$, $l_{4}=1.37 \AA \quad(\mathrm{CO}-\mathrm{NH}), \quad n_{5}=6, \quad l_{5}=1.50 \AA$ $\left(\Phi-\mathrm{CH}_{2}\right), n_{6}=6, l_{6}=1.43 \AA(\mathrm{NH}-\Phi), n_{7}=6$, $l_{7}=2.8 \AA(\Phi), n_{8}=2, l_{8}=1.43 \AA\left(\mathrm{NH}-\mathrm{CH}_{2}\right)$ and $m_{1}=173, M_{1}=14\left(\mathrm{CH}_{2}\right), m_{2}=8, M_{2}=$ $15(\mathrm{NH}), m_{3}=6, M_{3}=28(\mathrm{CO}), m_{4}=6, M_{4}$ $=16(\mathrm{O})$ and $m_{5}=6 \quad M_{6}=76(\Phi)$. Putting these values into eq 7 and 8 , we obtain $l=1.53 \mathrm{~A}$ and $\bar{M}_{\mathrm{b}}=16.1$ and then $A_{\mathrm{f}}$ was 


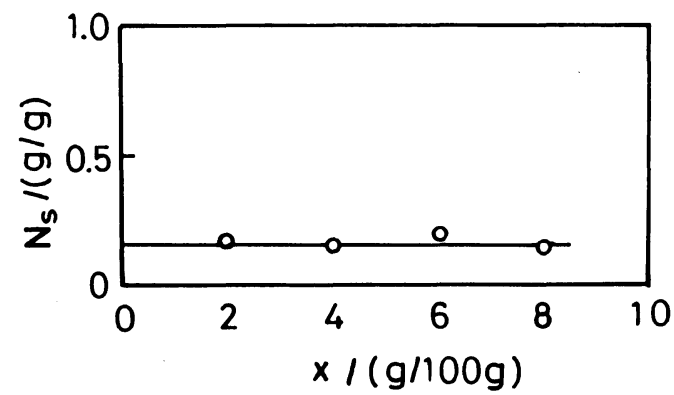

Figure 7. Plot of $N_{\mathrm{s}}$ versus concentration $x$ for the segment polyurethane in DMAc at $25^{\circ} \mathrm{C}$.

estimated to be $0.538 \times 10^{-8} \mathrm{~cm}$, here $\theta=$ $109^{\circ}$ was assumed. Then we can obtain the steric factor $\sigma$ of 1.86 and characteristic ratio $C_{\infty}$ of 6.88 . The segment polyurethane has a large chain flexibility and this may be attributed to the large flexibility of polytetramethylene glycol $(\sigma=1.6)^{16,17}$ which is a main portion $(\sim 80 \mathrm{wt} \%)$ of segment polyurethane.

Figure 7. shows the dependence of the weight of the solvated solvent per gram of polymer $N_{\mathrm{s}}$ on the polymer concentration $x(\mathrm{~g} / 100 \mathrm{~g}$ solvent $)$ of the segment polyurethane in DMAc at $25^{\circ} \mathrm{C} . N_{\mathrm{s}}$ was relatively small $(0.3 \mathrm{~g} / \mathrm{g})$ and nearly constant, irrespective of polymer concentration. This value means that on the average, a single bonding group among 20 bonding groups solvates with DMAc and the degree of solvation is relatively smaller than that for cellulose diacetate in DMAc $\left(N_{\mathrm{s}}=2\right) .{ }^{18} \mathrm{~A}$ more detailed specification of the bonding atoms solvated is beyond the scope of this study.

\section{REFERENCES}

1. H. Ono and I. Kimura, "Structure and Properties of Segment Polyurethane," Kagaku Dojin, Kyoto, 1971.

2. H. Sato, Bull. Chem. Soc. Jpn., 39, 2335 (1966).

3. D. J. Lyman, J. Polym. Sci., 45, 49 (1960).

4. H. C. Beachell and J. C. Peterson, J. Polym. Sci., A1, 7, 2021 (1969).

5. K. Kamide and M. Saito, Eur. Polym. J., 20, 903 (1984).

6. A. Passynsky, Acta Physicochim., USSR, 22, 137 (1947).

7. H. Okuto, Makromol. Chem., 98, 148 (1966).

8. A. Kaji and M. Murano, Polym. Prepr. Jpn., 33, 2219 (1984).

9. Y. Miyazaki and K. Kamide, to be submitted to Kobunshi Ronbunshu.

10. W. Bushuk and H. Benoit, Can. J. Chem., 36, 1616 (1958).

11. M. Kurata, M. Fukatsu, H. Sotobayashi, and H. Yamakawa, J. Chem. Phys., 41, 139 (1964).

12. M. Fixman, J. Chem. Phys., 36, 3123 (1962).

13. H. Baumann, J. Polym. Sci., Polym. Lett. Ed., 3, 1069 (1965).

14. W. H. Stockmayer and M. Fixman, J. Polym. Sci., C, 1, 137 (1963).

15. K. Kamide and W. Moore, J. Polym. Sci., Polym. Lett. Ed., 2, 1029 (1964).

16. M. Kurata, H. Utiyama, and K. Kamada, Makromol. Chem., 88, 281 (1965).

17. J. M. Evans and M. B. Huglin, Makromol. Chem., 127, 141 (1969).

18. K. Kamide and M. Saito, Eur. Polym. J., 20, 903 (1984). 